\title{
Prévention technique lors de travaux dans les bâtiments floqués à l'amiante
}

\author{
P. A. Cuendet, O. Schenk \\ Service neuchâtelois de médecine du travail et d'hygiène industrielle, Neuchâtel
}

Le Canton de Neuchâtel est en train de dresser l'inventaire de ses bastiments publics contenant des revêtements mous (flocages) à base d'amiante. Le but de cette enquête est de pouvoir contrôler, voire éliminer en toute sécurité de tels flocages sans que la santé des artisans ou des occupants du bâtiment soit mise en danger.

La première êtape dans un bâtiment suspect consiste à dëterminer la présence ou l'absence d'amiante dans les revêtements présents. Ces analyses d'échantillons de matêriaux d'isolation peuvent se faire à peu de frais à l'aide d'un microscope polarisant. Les différents types d'amiante sont rapidement identifiés en observant les effets de couleurs spécifiques et reproductibles engendrés par dispersion optique selon McCrone

(1). L'interprétation de telles observations est grandement facilitée par l'utilisation d'un atlas photomicrographique $(1,2)$.

En raison de leur dégradation, mais aussi pour cause de transformation ou de rénovation, des flocages doivent être arrachês. Un tel travail provoque la libêration de taux importants de poussières cancérigènes et implique donc la protection individuelle des travailleurs (3), l'étanchéité du chantier et la surveillance de la contamination. Cette surveillance s'effectue par mesure dans l'air des taux de microfibrilles libérées (4).

Depuis 1983, le Service neuchâtelois de médecine du travail et d'hygiène industrielle a contrôlé 4 chantiers d'arrachage de flocages à 1 'amiante. La technique de travail appliquée se résume en traitements par zones entièrement isolées du reste du bâtiment. La zone de travail est rendue ètanche à l'aide de feuilles de polyêthylène. On y accède par un sas comprenant 2 à 3 compartiments. Au coeur du système, il y a une machine qui se compose d'un séparateur de débris et d'un aspirateur de poussières muni d'un microfiltre (5). Les dêchets de flocage sont collectês à la source et recueillis dans des sacs étanches. L'air aspirê est dépoussiérê avant d'être rejeté à l'extêrieur. Par l'action de l'aspirateur, une dépression est créêe à l'intérieur du chantier; de cette manière, les poussières qui se dēgagent restent confinées dans la zone protégée. Le taux moyen de fibres libērées pendant 1 'arrachage est bas $(0.7$ fibre/ml). Il se situe en dessous de la valeur MAC qui est de 1 fibre/m) d'air (CNA, 1984). Le taux de contamination à l'extérieur du chantier a toujours été négligeable. En fin de travaux, les mesures effectuées dans les locaux dēcontaminés et nettoyẽs ont sans exception démontré l'absence de contamination résiduelle.
Summary :

Asbestos control programs in buildings start with the identification of the fibrous constituants in bulk samples. Polarized light microscopy with optical dispersion staining is a unique identification technique for asbestiform substances. Most asbestos control programs are undertaken to eliminate or minimize contamination from friable spray-applied coatings. The asbestos removal concept presented consists of stripping dry using a mobile vacuum-collector to prevent the spread of asbestos waste on the floor. Simultaneously the work area is maintained under a slight negative pressure to prevent airborne fibers from escaping.

\section{Références :}

(1) McCrone, W.C., The Asbestos Particle Atlas, Ann Arbor Science Publishers (1980).

(2) Mccrone, W.C. and Delly, The Particle Atlas, 2nd ed., Vol. Il (1974), Vol. V (1978), Ann Arbor Science Publishers.

(3) P.A. Cuendet et D. Bilat : Evaluation du degrê de protection d'un système masque-cartouche lors d'un travail avec de l'amiante. Méd. soc. prếv. 29 , 42-43 (1984).

(4) NIOSH Method 7400 , NIOSH Manual of Analytical Methods, 3rd ed., DHHS 84-100 (1984).

(5) Cuendet, P.A., Schweizer C., Brugger E. and Madelaine P. : New Method for Dry Removal of Asbestos Using a Commercially Available Vacuum Plant, Abstract \# 148, American Industrial Hygiene Conference, May 20-25, 1984, Detroit, USA.

\section{Adresse de correspondance :}

Dr. P.A. Cuendet, Service neuchatelois de médecine du travail et d'hygiène industrielle, Avenue de Bellevaux 51,2000 Neuchåtel. 